\title{
Endoscopic ultrasound-guided fine-needle aspiration plus KRAS and GNAS mutation in malignant intraductal papillary mucinous neoplasm of the pancreas
}

Authors

Institutions
Barbara Bournet ${ }^{1,2}$, Alix Vignolle-Vidoni ${ }^{2}$, David Grand ${ }^{3}$, Céline Roques ${ }^{3}$, Florence Breibach ${ }^{3}$, Jérome Cros $^{4}$, Fabrice Muscari ${ }^{5}$, Nicolas Carrère ${ }^{2,6}$, Janick Selves ${ }^{2,3}$, Pierre Cordelier ${ }^{2}$, Louis Buscail ${ }^{1,2}$

Institutions are listed at the end of article. submitted

20. February 2016

accepted after revision

19. August 2016

\section{Bibliography}

DOI http://dx.doi.org/

10.1055/s-0042-117216

Published online: 10.11.2016

Endoscopy International Open

2016; 04: E1228-E1235

(c) Georg Thieme Verlag KG

Stuttgart · New York

E-ISSN 2196-9736

\section{Corresponding author}

Louis Buscail, MD, PhD

Department of

Gastroenterology and INSERM

U1037

CHU Rangueil

1 avenue Jean Poulhès

TSA 50032

31059 Toulouse Cedex 9

France

Phone: +33 561323055

Fax: +33 561322229

Buscail.L@chu-toulouse.fr
Background: KRAS and GNAS mutations are common in intraductal papillary mucinous neoplasia of the pancreas (IPMN). The aims of this study were to assess the role of pre-therapeutic cytopathology combined with KRAS and GNAS mutation assays within cystic fluid sampled by endoscopic ultrasound-guided fine-needle aspiration (EUSFNA) to predict malignancy of IPMN.

Patients and methods: We prospectively included 37 IPMN patients with clinical and/or imaging predictors of malignancy (men: 24; mean age: 69.5 years). Cytopathology (performed on cystic fluid and/or IPMN nodules), KRAS (Exon 2, codon 12) and GNAS (Exon 8, codon 201) mutations assays (using TaqMan ${ }^{\circledR}$ allelic discrimination) were performed on EUS-FNA material. The final diagnosis was obtained from IPMN resections $(\mathrm{n}=$ 18); surgical biopsies, EUS-FNA analyses, and fol-

\section{Introduction}

$\nabla$

Intraductal papillary mucinous neoplasia of the pancreas (IPMN) is characterized by adenomatous proliferation of the pancreatic-duct epithelium and may involve the main pancreatic duct (MPD), the branch ducts (BD), or both 1). Accordingly, IPMNs are named after the structure from which they are derived and are classified into 3 groups: the MPD, branch duct, or mixed (when they originate from both the main duct and branch ducts).

Without exception, all cases of IPMN are considered to be potentially malignant $[1,2]$ and surgical resection of pancreatic lesions is recommended to prevent transformation into malignancy. In most cases, BD-IPMNs are benign, but there is a $15 \%$ risk of invasive carcinoma [3 -8]. Conversely, MPD- and mixed-IPMN cases are frequently malignant with a $50 \%$ risk of invasive carcinoma [9-12]. Until now, predictive factors for malignancy have not been fully defined and are still de- low-up ( $\mathrm{n}=19$ ): 10 and 27 IPMN were benign and malignant, respectively.

Results: Sensitivity, specificity, positive and negative predictive values, and accuracy of cytopathology alone to diagnose IPMN malignancy were $55 \%, 100 \%, 100 \%, 45 \%$, and $66 \%$, respectively. When KRAS-mutation analysis was combined with cytopathology these values were $92 \%, 50 \%$, $83 \%, 71 \%$, and $81 \%$, respectively. GNAS assays did not improve the performances of cytopathology alone or those of cytopathology plus a KRAS assay.

Conclusions: In patients with a likelihood of malignant IPMN at pre-therapeutic investigation, testing for KRAS mutations in cystic fluid sampling by EUS-FNA improved the results of cytopathology for the diagnosis of malignancy whereas GNAS mutation assay did not.

bated, even considering the consensus meeting held in 2012 [2].

Many studies, including ours, have provided several clinical and imaging pre-therapeutic criteria for malignancy, which greatly influence the prognosis and mortality from IPMN, such as age ( $>70$ years), presence of symptoms, a BD lesion with a diameter $>3 \mathrm{~cm}$, dilatation of the MPD, presence of mural solid nodules and/or thickening of a branch-duct cyst or the MPD wall, lymph nodes, peri-pancreatic extension, and positive cytology [11-14]. The recent consensus divided these indications into "high-risk stigmata" and "worrisome features" [2].

Taking into consideration that surgical resection of IPMN with a "malignancy signature" is the best-recommended treatment, the benefit-risk ratio should be considered. Surgical intervention may include major pancreatic resection, such as the Whipple procedure, or total pancreatectomy, as is routinely used for multifocal lesions: these procedures have risks of mortality and morbidity of $0 \%$ to $5 \%$ and $30 \%$ to $50 \%$, respectively [15]. 
Therefore, in aged patients and/or patients with comorbidities, a preoperative diagnosis or a prediction of malignancy could be highly useful in current clinical practice.

Endoscopic ultrasound (EUS) is a highly sensitive imaging modality used to evaluate pancreatic cystic lesions. Assessment of cyst fluid for carcinoembryonic antigen (CEA) levels does not differentiate IPMN from mucinous cystic neoplasm and does not correlate with the degree of dysplasia or malignancy [16-18].

Molecular pathology and genetic changes have been studied in IPMN and have shown that a point KRAS mutation is found in $48 \%$ of benign cases of IPMN (low-grade or intermediate dysplasia) $[19,20]$. GNAS mutations are present in $50 \%$ to $64 \%$ of cases of IPMN (predominantly of the intestinal subtype and to a lesser extent the gastric subtype) [21-24]. Recent studies report that GNAS and/or KRAS mutations were found in $90 \%$ of cases of IPMN $[24,25]$. However, the role of KRAS and GNAS assays for the diagnosis of malignancy remains controversial and preliminary, taking into account that mutations of KRAS and GNAS were found in $50 \%$ to $83 \%$ and $25 \%$ to $83 \%$ of malignant IPMN, respectively $[19,23,24]$. In other terms, whether DNA-based mutation assays are good biomarkers for predicting malignancy of IPMN remains to be demonstrated.

The aims of our study were to assess the role of pre-therapeutic cytopathology combined with KRAS and GNAS mutation assays within cystic fluid, sampled by EUS-FNA, to predict malignancy in a subgroup of patients with a likelihood of degenerative IPMN.

\section{Patients and methods}

\section{V}

\section{Patients and inclusion criteria}

Forty-one patients with BD, MPD-IPMN, or mixed-IPMN were prospectively enrolled between January 2010 and December 2014. A diagnosis of BD IPMN was established from radiologic criteria when unilocular or multilocular lesions with grapelike structures were observed communicating with the pancreatic ducts. In cases of mixed- or MPD-IPMN, MPD dilatation $\geq 5 \mathrm{~mm}$ was present in all patients. These imaging criteria were formerly identified (including communication between BD-cyst and MPD) from at least 2 morphologic examinations, including computed tomography (CT) scan, magnetic resonance cholangiopancreatography (MRCP), and EUS.

We selected jaundice, acute pancreatitis, diabetes, BD dilatation $>30 \mathrm{~mm}$, mural-tissue component or thickening (branch-duct cyst or MPD wall), MPD size $>10 \mathrm{~mm}$, and lymph nodes as predictors of malignancy (i.e., a high likelihood of degenerative IPMN) as previously described $[1,10,11,13]$.

We excluded patients with IPMN and without clinical and/or imaging predictors of malignancy, a pancreatic cystic tumor that was different from IPMN, a solid pancreatic tumor, evidence of metastasis or a non-resectable pancreatic cystic tumor, and a contraindication to undergo an EUS-FNA.

\section{Data, imaging, and EUS-FNA at inclusion}

The clinical data recorded were age, gender, medical history, circumstances of diagnosis, and symptoms related to IPMN. All patients underwent helical triple-phase CT examinations and MRCP was performed in $81 \%$ of patients $[8,11]$. EUS-FNA procedures were performed, as previously described $[8,11,26]$ (Olympus GF-UC140T echo-endoscope - Hamburg, Germany). Data recorded at imaging were maximal diameter of the MPD and branchduct cyst, the tissular component surrounding the MPD, mural modules and/or tissular thickening of the MPD and/or BD cyst wall (mainly at EUS), localization of BD lesions, and maximal diameter of the side-branch IPMN.

In each patient with predictor signs of malignancy, a fine-needle aspiration of cystic fluid was obtained using a Wilson Cook USN1 $22 \mathrm{G}$ needle biopsy (Limerick, Ireland). All the cystic fluid was systematically sampled (with a collapse of cystic walls) and placed in a dry sterile tube for cytologic analysis (a minimum of $1 \mathrm{~mL}$ ), except 500 to 750 microliters that was kept for molecular analyses. In cases where there was a mural nodule or a solid component within the IPMN, another FNA was systematically performed during the same procedure, using a different needle, in order to obtain tissue for a histologic microbiopsy (core biopsy). In cases where IPMN was resectioned, the pathology and IPMN histologic subtypes were assessed. The pathologic classification of IPMN was adopted in this work as follows: low-grade dysplasia, highgrade dysplasia, and invasive carcinoma [1,2]. We considered malignant IPMN as having high-grade dysplasia and invasive carcinoma. In patients where an IPMN resection was not performed, clinical and biological follow-up were systematically conducted at least every 6 months (hospitalization or consultation), together with abdominal imaging (MRCP or CT) performed every 6 months for the first year, and then annually. In cases of clinical and/or radiologic evolution of IPMN, another EUS-FNA was performed.

For all patients, decisions on management were systematically made during a multidisciplinary meeting dedicated to pancreatic disease and that included at least 2 gastrointestinal surgeons, 2 gastroenterologists, an anesthesiologist, a pathologist and a radiologist. The regional platform committee for somatic genetics of tumors in the Midi-Pyrénées approved the protocol for the KRAS and GNAS assays from the EUS-FNA samples. Informed written and signed consent for investigations was obtained from each patient and the research was conducted in full accordance with ethical principles outlined in the Declaration of Helsinki.

\section{KRAS and GNAS mutation assays}

The cystic fluid from IPMN was sampled using EUS-FNA: 500$700-\mu \mathrm{L}$ aliquots were placed in dry sterile 1 -mL Eppendorf tubes, and were rapidly frozen at $-20^{\circ} \mathrm{C}$ until DNA was extracted. Samples were then centrifuged for 10 minutes at $8000 \mathrm{rpm}$. DNA was extracted from the pellets using a QIAamp DNA micro kit (QIAGEN-Les Ulis, France). Nucleic acids were quantified using a Nanovue spectrophotometer (GE Healthcare, Buckinghamshire, UK).

To identify KRAS exon 2, codon-12 mutations, we performed a mutation-detection assay based on custom TaqMan ${ }^{\circledR} \mathrm{MGB}^{\mathrm{TM}}$ dual probes (KRAS codon-12 mutations: c.34G >C/p.G12R; c.35G $>$ A/p.G12D; c.35G > T/p.G12V). Polymerase chain reaction (PCR) sequences were detected using $20 \mathrm{ng}$ of genomic DNA, as previously described [27]. The remaining DNA was subsequently used to assess GNAS mutations.

To identify the GNAS exon 8, codon-201 mutations, we performed a mutation-detection assay based on custom TaqMan ${ }^{\circledR}$ MGB ${ }^{\mathrm{TM}}$ dual probes (GNAS codon-201 mutations: c.601C $>\mathrm{T} / \mathrm{p}$. R201C and c.602G > A/pR201H). Each probe incorporated a 5' reporter dye (VIC/FAM) specific to the WT or SNP sequence, and a 3' non-fluorescent quencher. Positive controls included DNA that was extracted from resected IPMN specimens and with known GNAS R201C and R201H mutations (sequencing). The negative control included DNA extracted from human pancreatic cancer cells (MiaPaca2 cells) with a known GNAS wild type [28]. Ampli- 


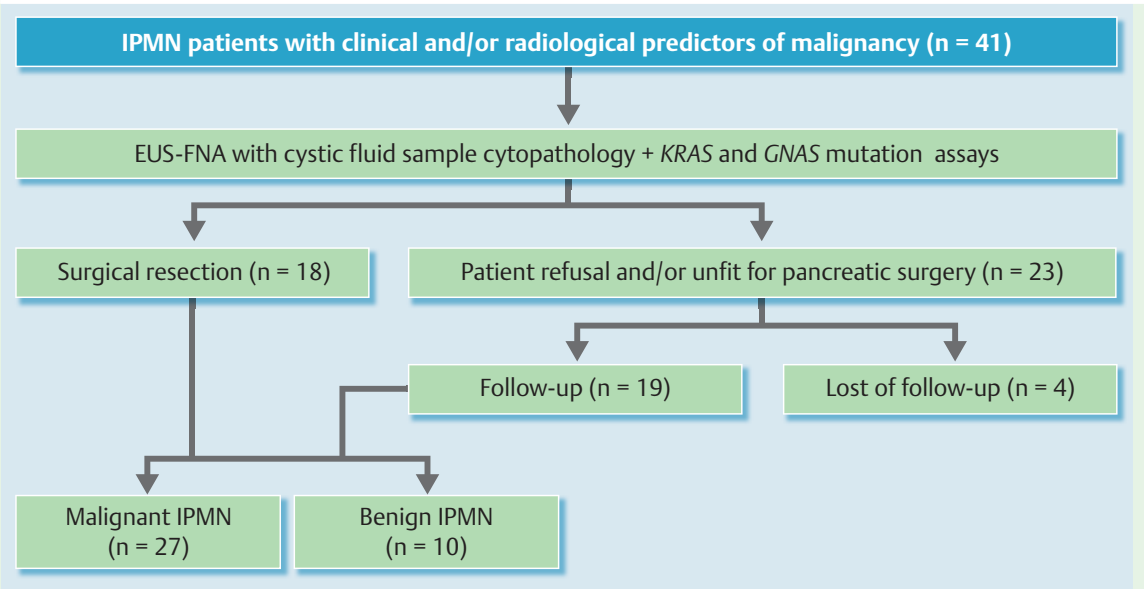

Fig. 1 Flowchart of the study, including distribution of intraductal papillary mucinous neoplasia of the pancreas (IPMN) in patients depending on resection or not and the follow-up. IPMN with predictors of malignancy that indicated a high likelihood of malignancy were: jaundice, branch-duct dilatation $>30 \mathrm{~mm}$, a mural-tissue component, dilatation of the main pancreatic duct $>10 \mathrm{~mm}$, or lymph nodes. Malignant IPMN means high-grade dysplasia and invasive carcinoma; benign IPMN means lowgrade dysplasia.

fication of the probe-specific product caused cleavage of the probe and generated an increase in reporter fluorescence. The use of a dual probe in each experiment made it possible to discriminate the signal for WT- and SNP-specific fluorescence in a single PCR and closed-tube format. Each alternation was screened with a dual-probe assay, with a wild-type and mutant for each of the 2 mutations screened. The runs were performed on a ROCHE LightCycler 480II (Roche Life Science, Boulogne-Billancourt, France) real-time PCR system. PCR was done in $10-\mu \mathrm{L}$ reaction volumes that included $20 \mathrm{ng}$ of genomic DNA and a $1 \mathrm{X}$ final master-mix custom Taqman ${ }^{\circledR}$ SNP genotyping assay (Life Technologies, Gent, Belgium). The cycling condition was a 3-step PCR in 96-well plates, as follows: denaturation at $95^{\circ} \mathrm{C}$ for $15 \mathrm{~min}$, amplification for 40 cycles at $95^{\circ} \mathrm{C}$ for $10 \mathrm{~s}$, at $60^{\circ} \mathrm{C}$ for $1 \mathrm{~min}$, and then cooling at $40^{\circ} \mathrm{C}$ for $30 \mathrm{~s}$. Each run included positive and negative controls. Investigators who performed the KRAS and GNAS assays were blinded to the diagnoses.

\section{Statistical analyses}

Results are expressed as mean \pm SE. Qualitative and quantitative data were analyzed using Student's $t$-test, the chi-squared test, or Fisher's exact test, and Spearman's and Pearson's correlation tests, as appropriate, using GraphPad-Instat (version 3.1a) and GraphPad-Prims (version 6) software.

\section{Results \\ $\nabla$}

\section{Patient characteristics and diagnoses}

Among the 41 patients diagnosed with IPMN, 18 underwent surgical resection with confirmation of IPMN in all cases (a Whipple procedure in 9, a left pancreatectomy in 7, a total pancreatectomy in 2). Twenty-three patients did not undergo resection because 3 cases of IPMN displayed signs of unresectability at laparotomy, 3 patients refused surgery, and 17 patients had contraindications for pancreatic surgery because of age and/or multiple comorbidities, including concomitant extra-pancreatic carcinoma.

In the non-surgical group, 4 patients refused follow-up, so a diagnosis could not be confirmed. Among the 41 patients included, 37 could be analyzed (the flowchart for the study is shown in - Fig. 1). Table 1 and Table 2 show the clinical, imaging, cytopathologic, and molecular characteristics at EUS-FNA of these 37 patients (men: 24; women: 13; mean age 69.5 years, median age 70 years). Half of the patients presented with at least 2 predictive signs of malignancy at diagnosis. No complication occurred during or after the EUS-FNA procedure. A final histologic diagnosis was obtained for all patients from resected specimens $(n=8)(-$ Table 1$)$. For non-resected IPMN the final diagnosis was obtained ( $\bullet$ Table 2 ): from biopsy tissue material obtained during a laparotomy $(n=3)$, from core biopsy at initial EUSFNA ( $n=12$ ) and/or subsequent follow-up with second biopsy (EUS- or CT-guided) and occurrence of metastasis in case of malignant IPMN. On the whole, all 19 patients with non-resected IPMN were followed up after diagnosis (median 13 months, range 4-60). In 8 patients with invasive carcinoma or high-grade dysplasia at baseline FNA $(n=6)$, or who had a surgical biopsy during laparotomy $(n=2)$ disease progressed both clinically and anatomically (median follow-up 7.5 months, deaths $n=5$ ). In the 6 patients with no malignancy found on EUS-FNA (low-grade dysplasia or normal/hyperplasia), an invasive carcinoma was subsequently diagnosed based on clinical and/or imaging evidence (including a subsequent EUS-guided or CT-guided FNA with histologic analysis of a microbiopsy as well as a surgical biopsy during laparotomy): the median time to diagnosis of carcinoma was 8.5 months (range 2-24) and there were 4 deaths. The 5 remaining patients did not have disease progression (median follow-up 52 months: range 14-58 months), and this included 3 subsequent EUS-FNAs that still showed low-grade dysplasia. In these 5 patients, a diagnosis of benign IPMN was definitively attributed. Taking into account resected and non-resected IMPN, the final diagnoses were benign IPMN in 10 and malignant IPMN in 27 patients (i.e., 2with high-grade dysplasia; 25 with invasive carcinoma).

\section{Cytopathology and yield of KRAS and GNAS assays at the} baseline EUS-FNA

Cytology and/or histologic analysis of EUS-FNA materials are detailed in Table 1 and Table 2 for patients with confirmed IPMN and carcinoma ( $n=11,30 \%)$, for IPMN with high-grade dysplasia ( $n=4,11 \%$ ), and for IPMN with low-grade dysplasia and normal/hyperplasia ( $\mathrm{n}=22,59 \%)$. KRAS and a GNAS assays were possible in all cases except one because of insufficient DNA material for subsequent GNAS-mutation analysis after assessing KRAS status. Fig. 2 shows the representative curve plots for wild-type and mutated KRAS and GNAS samples. The mean quantity of DNA extracted per sample was $269 \pm 58 \mathrm{ng}$ (min: $35 \mathrm{ng}$; max: $1660 \mathrm{ng}$ ). There was no statistical difference in terms of quantity of DNA extracted from the subgroups with wild-type $(232 \pm 73 \mathrm{ng})$ or mutated ( $290 \pm 86 \mathrm{ng})$ KRAS and GNAS $(P=0.65)$, or between the 2 subgroups with malignant $(250 \pm 78 \mathrm{ng})$ or be- 
Table 1 Clinical, anatomical, and molecular characteristics, and pathologic findings in patients with resected branch-duct (BD)-, mixed-, or main pancreatic duct (MPD) intraductal papillary mucinous neoplasia of the pancreas (IPMN).

\begin{tabular}{|c|c|c|c|c|c|c|c|c|c|c|c|}
\hline$\#$ & Sex & Age & signs & Type & $\begin{array}{l}\text { BD size } \\
(\mathrm{mm})\end{array}$ & $\begin{array}{l}\text { MPD size } \\
(\mathrm{mm})\end{array}$ & Site & $\begin{array}{l}\text { Cytopatholo- } \\
\text { gy } \\
\text { at EUS-FNA }\end{array}$ & $\begin{array}{l}\text { KRAS } \\
\text { status }\end{array}$ & $\begin{array}{l}\text { GNAS } \\
\text { status }\end{array}$ & $\begin{array}{l}\text { Final histology } \\
\text { and diagnosis }\end{array}$ \\
\hline 1 & M & 62 & Pain & Mixed & 27 & 6 & Head & Normal & G12D & R201C & IC, gastric \\
\hline 2 & W & 75 & None & Mixed & 60 & 8 & Head & Carcinoma & WT & WT & HGD pancreato-biliary \\
\hline 3 & M & 58 & Pain & Mixed & 32 & 11 & Head & Carcinoma & G12V & WT & IC, intestinal \\
\hline 4 & W & 76 & Diabetes & $\mathrm{BD}$ & 35 & 3.5 & Body & Carcinoma & G12V & WT & IC, intestinal \\
\hline 5 & W & 71 & None & Mixed & 6 & 12 & Head & Normal & WT & WT & IC, intestinal \\
\hline 6 & $M$ & 72 & Pain & Mixed & 70 & 8 & Head & Carcinoma & WT & WT & IC, intestinal \\
\hline 7 & M & 59 & AP & Mixed & 35 & 7 & Head & Carcinoma & WT & WT & IC intestinal \\
\hline 8 & M & 65 & None & Mixed & 25 & 6 & Body & Carcinoma & G12V & WT & IC, intestinal \\
\hline 9 & W & 80 & None & Mixed & 50 & 6 & Tail & LGD & G12D & WT & HGD, gastric \\
\hline 10 & M & 57 & None & Mixed & 40 & 8 & head & LGD & WT & WT & LGD, gastric \\
\hline 11 & M & 61 & AP & Mixed & 40 & 5 & head & Normal & G12D & $\mathrm{R} 201 \mathrm{H}$ & LGD, gastric \\
\hline 12 & W & 72 & Weight loss & $\mathrm{BD}$ & 90 & 5 & head & HGD & WT & $\mathrm{R} 201 \mathrm{H}$ & IC, gastric \\
\hline 13 & W & 66 & None & Mixed & 18 & 6 & body & Carcinoma & WT & WT & IC, intestinal \\
\hline 14 & M & 65 & none & Mixed & 33 & 6 & head & Normal & G12V & $\mathrm{R} 201 \mathrm{H}$ & IC, gastric \\
\hline 15 & M & 68 & Weight loss & Mixed & 30 & 7 & head & LGD & G12D & R201C & LGD, intestinal \\
\hline 16 & M & 77 & Pain & Mixed & 32 & 12 & body & Normal & G12D & WT & LGD, gastric \\
\hline 17 & M & 62 & Pain & Mixed & 40 & 9 & head & LGD & G12D & WT & IC, pancreato-biliary \\
\hline 18 & M & 70 & AP & $\mathrm{BD}$ & 25 & 3 & head & LGD & G12D & R201C & LGD, gastric \\
\hline
\end{tabular}

IC: IPMN with invasive adenocarcinoma; HGD = high-grade dysplasia IPMN; LGD=intermediate or low-grade dysplasia IPMN; AP: acute pancreatitis; BD: branch duct IPMN; MD: main-duct IPMN; mixed: mixed IPMN. WT: wild-type.

nign $(288 \pm 103 \mathrm{ng})$ IPMN at the final diagnosis $(P=0.85)$ (unpaired Student's $t$ test). In addition, there was no correlation between the size of the BD-IPMN in which fluid samples had been made by EUS and the amount of extracted DNA (Pearson's and Spearman's correlation tests: $0.027<\mathrm{r}<0.05$, data not shown). This indicates that neither the presence of malignancy nor the size of the side-branch IPMN influenced the amount of DNA in the cystic fluid.

\section{Combination of cytopathology and the KRAS/GNAS mutation assays to predict IPMN malignancy}

Data on sensitivity, specificity, predictive values, overall accuracy of the cytopathology alone, the KRAS analysis alone, the GNAS analysis alone, and the combination of either 2 or 3 tests to diagnose malignant versus benign IPMN are detailed in $\bullet$ Table 3 .

We observed that combining cytopathologic analysis and the KRAS mutation assay increased the sensitivity (from 53 to $92 \%$ ), the negative predictive value (NPV) (from 45 to $71 \%$ ), and the accuracy (from 66 to $81 \%$ ) compared to cytopathology alone to diagnose malignant IPMN. This was not observed when using the combined cytopathologic analysis and GNAS-mutation assay. In addition, combining the cytopathology and KRAS and GNAS assays did not improve the performance of combining cytopathology and the KRAS assay. In $\odot$ Table 4 we compared the performances of cytopathology alone and the combination of cytopathology plus KRAS status in the 2 subgroups of patients that did or did not undergo resection of their IPMN. In these 2 subgroups, the sensitivity, the NPV, and the accuracy of cytopathology alone to diagnose IPMN malignancy were also increased when they were combined with the cystic fluid KRAS-mutation assay. Overall, we can conclude that when the EUS-FNA cytopathology and KRAS mutation assay are combined we can predict a malignancy in $80 \%$ of cases of IPMN.

\section{Discussion}

$\nabla$

In this prospective study, we investigated a subgroup of IPMN patients who had clinical predictors of malignancy assessed at diagnosis. We demonstrated that when the KRAS-mutation analysis was added to the cytopathology data from EUS-FNA materials, this improved the ability to diagnose malignancy. This is of importance as it will improve our ability to identify patients with high-grade dysplasia or invasive malignant IPMN and who might benefit from surgical resection. We also observed that testing for the GNAS mutation did not improve the performance of cytopathology alone or the combination of cytopathology plus the KRAS assay to diagnose this indication.

As previously observed in solid tumors, despite the paucity of material collected for molecular analysis from IPMN cystic fluid, which rarely excesses $500 \mu l$, DNA extraction can be performed in $100 \%$ of cases [27]. Use of an extraction micro-kit enables this success rate. The amount of DNA was half that observed from EUS-FNA materials from solid pancreatic tumors [27]. In addition, the amount of DNA was not influenced by the mutational status, the size of the cyst or its stage. To our knowledge, this study demonstrates, for the first time, that TaqMan allelic discrimination is feasible on material collected from pancreatic cystic fluid to assess both KRAS and GNAS mutation status.

As already observed, cytopathology has a sensitivity of $~ 50 \%$ (with high specificity) in in diagnosing malignancy of IPMN [16-18] but it is inferior to pre-therapeutic clinical imaging. In comparison, the codon-12 KRAS mutation assay alone had performance similar to that of cytopathology, with lower specificity and positive predictive value due to false-positive results (i.e., the presence of KRAS mutations in patients with low-grade dysplasia). However, when cytopathology and the KRAS assay are combined, sensitivity, NPV, and accuracy were substantially increased (sensitivity increased from 55 to $92 \%$, NPV from $45 \%$ to $71 \%$, and accuracy from $66 \%$ to $81 \%$ ). This indicates that associating cytopathology and testing KRAS in EUS-FNA material from 
Table 2 Clinical, anatomic, and molecular characteristics, and pathologic findings of patients with non-resected branch-duct (BD)-, mixed-, or main pancreatic duct (MPD) intraductal papillary mucinous neoplasia of the pancreas (IPMN).

\begin{tabular}{|c|c|c|c|c|c|c|c|c|c|c|c|}
\hline \#. & Gender & Age & Signs & Type & $\begin{array}{l}\text { BD size } \\
(\mathrm{mm})\end{array}$ & $\begin{array}{l}\text { MPD size } \\
(\mathrm{mm})\end{array}$ & Site & $\begin{array}{l}\text { Cytopathology } \\
\text { at EUS-FNA }\end{array}$ & $\begin{array}{l}\text { KRAS } \\
\text { status }\end{array}$ & GNAS status & $\begin{array}{l}\text { Final diagnosis } \\
\text { (assessment) }\end{array}$ \\
\hline 1 & W & 56 & Pain & $\mathrm{BD}$ & 30 & 4 & Head & $\begin{array}{l}\text { Carcinoma } \\
\text { (cytology) }\end{array}$ & G12D & WT & $\begin{array}{l}\text { IC, gastric } \\
\text { (surgical biopsy) }\end{array}$ \\
\hline 2 & M & 73 & Pain & $\mathrm{BD}$ & 50 & 4 & Head & $\begin{array}{l}\text { Normal } \\
\text { (cytology) }\end{array}$ & G12D & WT & $\begin{array}{l}\text { IC, intestinal } \\
\text { (surgical biopsy) }\end{array}$ \\
\hline 3 & $\mathrm{M}$ & 75 & Pain & $\mathrm{BD}$ & 60 & 4 & Body & $\begin{array}{l}\text { HGD } \\
\text { (core biopsy) }\end{array}$ & WT & R201C & $\begin{array}{l}\text { IC } \\
\text { (surgical biopsy) }\end{array}$ \\
\hline 4 & W & 82 & Jaundice & Mixed & 20 & 12 & Body & $\begin{array}{l}\text { HGD } \\
\text { (core biopsy) }\end{array}$ & G12D & WT & $\begin{array}{l}\text { IC } \\
\text { (follow-up) }\end{array}$ \\
\hline 5 & $\mathrm{M}$ & 79 & $\begin{array}{l}\text { Weight } \\
\text { loss }\end{array}$ & Mixed & 30 & 7 & Head & $\begin{array}{l}\text { LGD } \\
\text { (core biopsy) }\end{array}$ & G12V & WT & $\begin{array}{l}\text { IC } \\
\text { (follow-up) }\end{array}$ \\
\hline 6 & $\mathrm{M}$ & 64 & None & Mixed & 33 & 8 & Head & $\begin{array}{l}\text { LGD } \\
\text { (core biopsy) }\end{array}$ & G12V & WT & $\begin{array}{l}\text { LGD } \\
\text { (second biopsy) }\end{array}$ \\
\hline 7 & $\mathrm{M}$ & 73 & Jaundice & $\mathrm{BD}$ & 35 & 3.5 & Head & $\begin{array}{l}\text { HGD } \\
\text { (core biopsy) }\end{array}$ & G12D & WT & $\begin{array}{l}\text { IC, intestinal } \\
\text { (follow-up) }\end{array}$ \\
\hline 8 & M & 78 & Diabetes & $\mathrm{BD}$ & 32 & 5 & Head & $\begin{array}{l}\text { LGD } \\
\text { (cytology) }\end{array}$ & WT & WT & $\begin{array}{l}\text { LGD } \\
\text { (follow-up) }\end{array}$ \\
\hline 9 & $\mathrm{M}$ & 67 & None & Mixed & 35 & 7 & Head & $\begin{array}{l}\text { Normal } \\
\text { (cytology) }\end{array}$ & G12D & WT & $\begin{array}{l}\text { IC } \\
\text { (follow-up) }\end{array}$ \\
\hline 10 & M & 85 & Jaundice & Mixed & 40 & 8 & Head & $\begin{array}{l}\text { Normal } \\
\text { (cytology) }\end{array}$ & G12V & WT & $\begin{array}{l}\text { IC } \\
\text { (follow-up) }\end{array}$ \\
\hline 11 & W & 57 & Pain & Mixed & 75 & 6 & Body & $\begin{array}{l}\text { Normal } \\
\text { (cytology) }\end{array}$ & G12D & WT & $\begin{array}{l}\text { IC } \\
\text { (second biopsy) }\end{array}$ \\
\hline 12 & W & 58 & None & $\mathrm{BD}$ & 50 & 3 & Body & $\begin{array}{l}\text { LGD } \\
\text { (core biopsy) }\end{array}$ & WT & WT & $\begin{array}{l}\text { LGD } \\
\text { (follow-up) }\end{array}$ \\
\hline 13 & W & 67 & Pain & $\mathrm{BD}$ & 30 & 4 & Body & $\begin{array}{l}\text { HGD } \\
\text { (core biopsy) }\end{array}$ & G12R & WT & $\begin{array}{l}\text { IC } \\
\text { (follow-up) }\end{array}$ \\
\hline 14 & W & 78 & None & $\mathrm{BD}$ & 31 & 3 & Body & $\begin{array}{l}\text { LGD } \\
\text { (core biopsy) }\end{array}$ & WT & WT & $\begin{array}{l}\text { LGD } \\
\text { (follow-up) }\end{array}$ \\
\hline 15 & M & 64 & Jaundice & Mixed & 33 & 8 & Head & $\begin{array}{l}\text { Carcinoma } \\
\text { (core biopsy) }\end{array}$ & WT & R201C & $\begin{array}{l}\text { IC, intestinal } \\
\text { (follow-up) }\end{array}$ \\
\hline 16 & W & 70 & Jaundice & Mixed & 30 & 5 & Head & $\begin{array}{l}\text { LGD } \\
\text { (cytology) }\end{array}$ & WT & WT & $\begin{array}{l}\text { IC } \\
\text { (follow-up) }\end{array}$ \\
\hline 17 & M & 84 & Pain & Mixed & 60 & 6 & Head & $\begin{array}{l}\text { Normal } \\
\text { (core biopsy) }\end{array}$ & WT & WT & $\begin{array}{l}\text { LGD } \\
\text { (second biopsy) }\end{array}$ \\
\hline 18 & M & 67 & $\begin{array}{l}\text { Bowel ob- } \\
\text { struct. }\end{array}$ & Mixed & 35 & 11 & Body & $\begin{array}{l}\text { Carcinoma } \\
\text { (core biopsy) }\end{array}$ & G12D & WT & $\begin{array}{l}\text { IC, gastric } \\
\text { (follow-up) }\end{array}$ \\
\hline 19 & M & 78 & None & Mixed & 28 & 7 & Head & $\begin{array}{l}\text { Carcinoma } \\
\text { (core biopsy) }\end{array}$ & G12D & ND & $\begin{array}{l}\text { IC } \\
\text { (follow-up) }\end{array}$ \\
\hline
\end{tabular}

IC: IPMN with invasive adenocarcinoma; HGD = high-grade dysplasia IPMN; LGD=intermediate or low-grade dysplasia IPMN; AP: acute pancreatitis; BD: branch duct IPMN; MD: main-duct IPMN; mixed: mixed IPMN. WT: wild-type. For initial EUS-cytopathology the material obtained is detailed in parenthesis: cytology alone (cytology $\mathrm{n}=7$ ) or histology on core biopsies (core biopsy $\mathrm{n}=12$ ). For the final diagnosis the assessment is detailed in parenthesis: surgical biopsy during laparotomy, second biopsy (EUS- or CT-guided) and follow-up (subsequent occurrence of metastasis).

IPMN may help predict malignancy in patients with BD or a mixed form of IPMN

Conversely, the codon 201 GNAS mutation assay did not improve diagnosis. Moreover GNAS mutation (22\%, intestinal and gastric subtypes) appeared less frequently than previously observed in micro-dissected specimens of resected IPMN, in cystic fluid, or in duodenal fluid sampled under secretin stimulation $(50 \%-66 \%$ of mutated GNAS) $[21,24,25,29]$. Interestingly, when considering the subgroup of IPMN with high-grade dysplasia and invasive carcinoma, frequency of GNAS mutations ranges from $15 \%-33 \%$ and is found to be significantly less in cases of IPMN with invasive carcinoma when compared to other histopathologic grades $[24,25$, 30]. Regarding the current study, almost $75 \%$ of patients with IMPN were diagnosed with high-grade dysplasia and invasive carcinoma. Such a patient population may account for the low frequency of GNAS mutations in our study.
A recent study from Tan et al., performed on micro-dissected IPMN tissues, did not find any significant difference in terms of KRAS or GNAS mutations according to the degree of dysplasia [22]. However, GNAS mutations were more frequent in colloidaltype (89\%) than in tubular-type (32\%) invasive carcinoma, respectively [22]. Conversely, KRAS mutations were significantly more frequent in the tubular-subtype ( $89 \%$ vs. $52 \%$ ). In our work, almost $50 \%$ of cases with resected IPMN and invasive carcinoma were either gastric or pancreatobiliary subtypes that generated adenocarcinoma with a tubular shape. Such an observation may also explain the low incidence of GNAS mutations in our series. Another explanation could be that cystic fluid sampled by EUSFNA may contain only neoplastic cells from a limited area and does not reflect the entire pancreas, including the MPD. However, the frequency of KRAS mutations in our patient cohort was $62 \%$, an amount that is commonly found in IPMN tissues and fluid, including within high-grade or invasive cases [23-25]. 


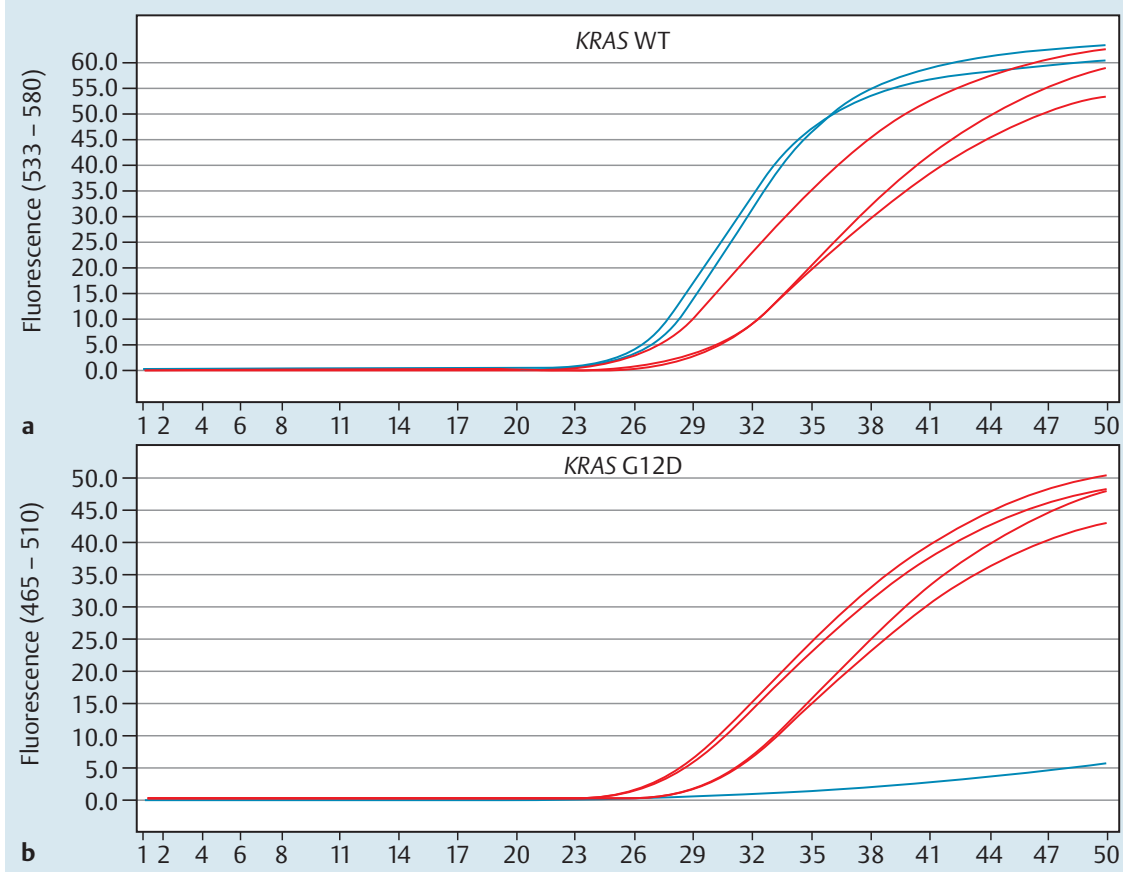

Fig.2 Representative chromatogram of TaqMan allelic discrimination analysis of G12D KRAS and R201C GNAS mutations obtained from EUS-guided fine-needle cystic-fluid aspiration from intraductal papillary mucinous neoplasia of the pancreas (IPMN). Panels a and $\mathbf{c}$ : plots of a wild-type DNA sample; panels $\mathbf{b}$ and $\mathbf{d}$ : plots of mutated DNA samples with distinct curves generated by mutated and wild-type probes.

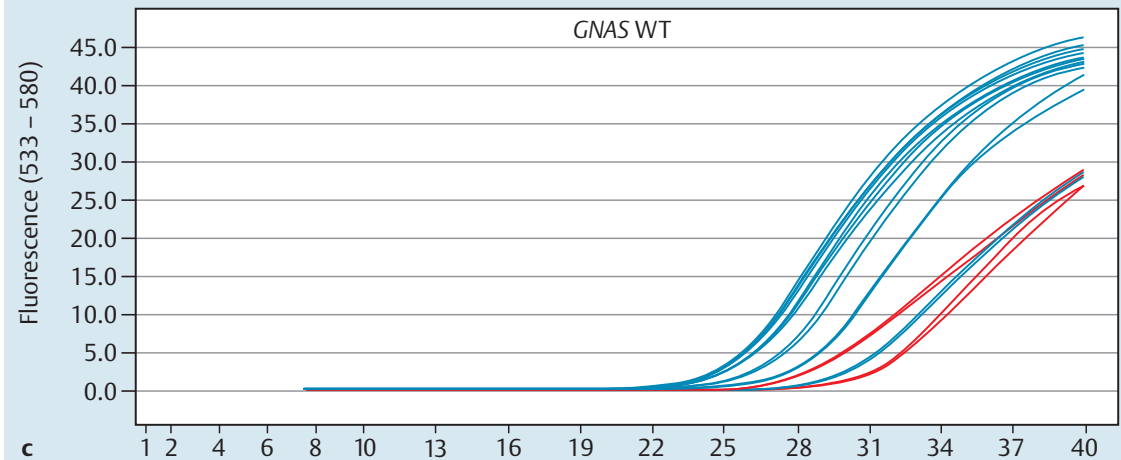

c

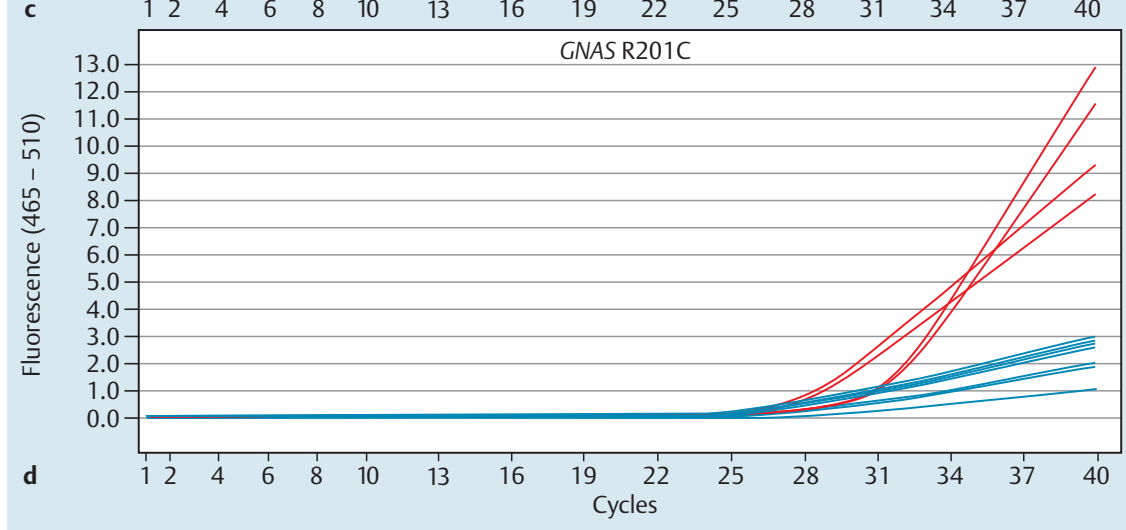

Table 3 Performance of cystic fluid EUS-FNA, EUS-FNA and a KRAS assay, EUS-FNA and a GNAS assay, EUS-FNA and a KRAS plus a GNAS assay to diagnose malignant intraductal papillary mucinous neoplasia of the pancreas (IPMN) in 37 patients with clinical and/or radiologic predictors of malignancy.

\begin{tabular}{|c|c|c|c|c|c|}
\hline & Sensitivity (95\% Cl) & Specificity (95\% Cl) & PPV (95\% CI) & NPV (95\% Cl) & Accuracy (95\% Cl) \\
\hline EUS-FNA & $55(35-94)$ & $100(69-100)$ & $100(78-100)$ & $45(24-67)$ & $66(54-96)$ \\
\hline KRAS & $66(46-83)$ & $50(18-80)$ & $78(56-92)$ & $36(12-64)$ & $61(48-92)$ \\
\hline GNAS & $19(6-38)$ & $70(34-93)$ & $62(24-91)$ & $24(10-43)$ & $61(43-87)$ \\
\hline EUS-FNA + KRAS & $92(75-99)$ & $50(18-81)$ & $83(66-93)$ & $71(29-96)$ & $81(67-96)$ \\
\hline EUS-FNA + GNAS & $62(42-80)$ & $70(34-93)$ & $85(62-96)$ & $41(18-67)$ & $64(37-83)$ \\
\hline EUS-FNA + KRAS+ GNAS & $92(75-99)$ & $50(18-81)$ & $83(66-93)$ & $71(29-96)$ & $81(67-96)$ \\
\hline
\end{tabular}

PPV: positive predictive value; NPV: negative predictive value; EUS-FNA: endoscopic ultrasound-fine-needle guided aspiration. 
Table 4 Performance of cystic fluid EUS-FNA and EUS-FNA plus a KRAS assay to diagnose malignant intraductal papillary mucinous neoplasia of the pancreas (IPMN) in two subgroups of patients with and without resection of IPMN and pre-therapeutic clinical and/or radiologic predictors of malignancy.

\begin{tabular}{|c|c|c|c|c|c|}
\hline & Sensitivity (95\% Cl) & Specificity (95\% Cl) & PPV (95\% Cl) & NPV $(95 \%$ Cl) & Accuracy $(95 \% \mathrm{Cl})$ \\
\hline \multicolumn{6}{|c|}{ Resected IPMN $(n=18)$} \\
\hline EUS-FNA & $61(31-86)$ & $100(47-100)$ & $100(63-100)$ & $50(18-81)$ & $72(48-91)$ \\
\hline EUS-FNA + KRAS & $91(61-99)$ & $33(4-77)$ & $73(44-92)$ & $66(9-99)$ & $72(43-99)$ \\
\hline \multicolumn{6}{|c|}{ Non-resected IPMN $(n=19)$} \\
\hline EUS-FNA & $50(23-76)$ & $100(47-100)$ & $100(59-100)$ & $42(15-72)$ & $63(45-87)$ \\
\hline EUS-FNA + KRAS & $78(49-95)$ & $80(28-99)$ & $92(61-99)$ & $57(18-90)$ & $79(52-99)$ \\
\hline
\end{tabular}

PPV: positive predictive value; NPV: negative predictive value; EUS-FNA: endoscopic ultrasound-fine-needle guided aspiration. There is no statistical difference in term of sensitivity and specificity between the two groups (resected versus non-resected $-0.65<P<0.73$, Fischer's exact test) except for the specificity of EUS-FNA + KRAS that was statistically lower in the resected group $(P=0.002)$.

From a clinical point of view, allelic discrimination assays for KRAS mutations are currently performed on somatic genetic tumors in colon cancer specimens, thus this approach now can be readily transferred into routine clinical practice for pre-therapeutic evaluation of IPMN in EUS-FNA material (and recently Next Generation Sequencing). When treating patients with high-risk of stigmata, EUS-FNA associated with a KRAS mutation assay could be useful when making therapeutic decisions [30]. From our results, in cases of high-grade dysplasia, combining cytopathology and/or KRAS-mutation assessment enabled prediction of malignancy in $80 \%$ of cases. Recent studies have underscored the importance of combining both clinical and radiologic findings to assessment of hotspots mutations of pancreatic cyst fluid for detection of advanced neoplasia, especially IPMN [31, 32].

From a practical point of view, payment for additional molecular analysis can be an issue. In our study, only 9 of 37 patients (24\%) had a normal or LGD cytology at EUS with mutated KRAS and a final diagnosis of invasive carcinoma arising in the IPMN. Therefore, we would suggest reserving KRAS mutation analysis for cases with normal or non-contributive or LGD cytopathology (keeping thus a minimum 500 microliters of cystic fluid to a possible subsequent molecular analysis).

In addition, following the international consensus for IPMPN, Crippa et al. recently reported some significant predictor signs of disease-specific survival after multivariate analysis. They were age $>70$ years, presence of atypical or malignant cytology, jaundice, and main pancreatic duct size $>15 \mathrm{~mm}[2,33]$. These data are important from a clinical point of view and, in our series, $54 \%$ of patients (20 over 37 ) had at least 1 of these criteria. Besides the results of cytopathology after EUS-FNA, some of these criteria should be taken into account and finally KRAS mutation analysis also can be reserved for patients who meet longer survival criteria.

\section{Conclusion \\ $\nabla$}

In conclusion, in patients with a pre-therapeutic likelihood of IPMN-associated malignancy, use of EUS-FNA to obtain cystic fluid was safe, and the TaqMan allelic discrimination assay for KRAS and GNAS mutations was feasible. Although testing for GNAS mutations did not substantially improve diagnosis IPMN malignancy, the KRAS mutation assay, when combined with cytopathology, increased performance of cytopathology alone, especially in sensitivity, NPV, accuracy, and as a predictor of malignancy in $80 \%$ of cases.
Competing interests: Financial support received from STIC Program Inca 2008, Inca PRK 2012 and gifts from A. C., M-H. C. and L. G.

\section{Institutions}

1 Department of Gastroenterology and INSERM UMR 1037, CHU Toulouse Rangueil, University of Toulouse, Toulouse, France

2 INSERM UMR 1037, University Institute of Cancer of Toulouse, University of Toulouse, Toulouse, France

${ }^{3}$ Department of Pathology, University Institute of Cancer of Toulouse, University of Toulouse, Toulouse, France

${ }^{4}$ Department of Pathology, Beaujon Hospital, University of Paris Diderot, Clichy, France

5 Department of Digestive Surgery, CHU Toulouse Rangueil, University of Toulouse, Toulouse, France

${ }^{6}$ Department of Digestive Surgery, CHU Toulouse Purpan, University of Toulouse, Toulouse, France

\section{References}

1 Tanaka M, Chari S, Adsay Vet al. International consensus guidelines for management of intraductal papillary mucinous neoplasms and mucinous cystic neoplasms of the pancreas. Pancreatology 2006; 6: 17-32

2 Tanaka M, Fernández-del Castillo C, Adsay Vet al. International consensus guidelines 2012 for the management of IPMN and MCN of the pancreas. Pancreatology 2012; 12: 183-197

3 Nagai K, Doi R, Kida A et al. Intraductal papillary mucinous neoplasms of the pancreas: clinicopathologic characteristics and long-term follow-up after resection. World J Surg 2008; 32: 271 - 278

4 Sadakari $Y$, Ienaga J, Kobayashi $K$ et al. Cyst size indicates malignant transformation in branch duct intraductal papillary mucinous neoplasm of the pancreas without mural nodules. Pancreas 2010; 39 : $232-236$

5 Kanno A, Satoh K, Hirota $M$ et al. Prediction of invasive carcinoma in branch type intraductal papillary mucinous neoplasms of the pancreas. J Gastroenterol 2010; 45: 952 -959

6 Hwang DW, Jang JY, Lee SE et al. Clinicopathologic analysis of surgically proven intraductal papillary mucinous neoplasms of the pancreas in SNUH: a 15-year experience at a single academic institution. Langenbecks Arch Surg 2012; 397: 93-102

7 Mimura T, Masuda A, Matsumoto I et al. Predictors of malignant intraductal papillary mucinous neoplasm of the pancreas. J Clin Gastroenterol 2010; 44: e224-229

8 Arlix A, Bournet B, Otal $P$ et al. Long-term clinical and imaging followup of nonoperated branch duct form of intraductal papillary mucinous neoplasms of the pancreas. Pancreas 2012; 41: 295-301

9 Anand $N$, Sampath $K, W u$ BU. Cyst features and risk of malignancy in itraductal papillary mucinous neoplasms of the pancreas: a meta-analysis. Clin Gastroenterol Hepatol 2013; 11: 913-921

10 Sugiyama $M$, Izumisato $Y$, Abe $N$ et al. Predictive factors for malignancy in intraductal papillary-mucinous tumours of the pancreas. Br J Surg 2003; 90: $1244-1249$

11 Bournet B, Kirzin S, Carrère $N$ et al. Clinical fate of branch duct and mixed forms of intraductal papillary mucinous neoplasia of the pancreas. J Gastroenterol Hepatol 2009; 24: $1211-1217$

12 Daudé M, Muscari F, Buscail C et al. Outcomes of nonresected mainduct intraductal papillary mucinous neoplasms of the pancreas. World J Gastroenterol 2015; 21: $2658-2667$ 
13 Salvia R, Fernández-del Castillo C, Bassi C et al. Main-duct intraductal papillary mucinous neoplasms of the pancreas: clinical predictors of malignancy and long-term survival following resection. Ann Surg 2004; 239: 678-685

14 Ogura T, Masuda D, Kurisu Y et al. Potential predictors of disease progression for main-duct intraductal papillary mucinous neoplasms of the pancreas. J Gastroenterol Hepatol 2013; 28: 1782-1786

15 Wang $H$, Chen T, Wang $H$ et al. A systematic review of the Physiological and Operative Severity Score for the enUmeration of Mortality and morbidity and its Portsmouth modification as predictors of post-operative morbidity and mortality in patients undergoing pancreatic surgery. Am J Surg 2013; 205: 466-472

16 Rockacy M, Khalid A. Update on pancreatic cyst fluid analysis. Ann Gastroenterol 2013; 26: 122-127

17 Suzuki R, Thosani N, Annangi S et al. Diagnostic yield of EUS-FNA-based cytology distinguishing malignant and benign IPMNs: a systematic review and meta-analysis. Pancreatology 2014; 14: 380-384

18 Maker AV, Carrara S, Jamieson NB et al. Cyst fluid biomarkers for intraductal papillary mucinous neoplasms of the pancreas: a critical review from the international expert meeting on pancreatic branch-duct-intraductal papillary mucinous neoplasms. J Am Coll Surg 2015; 220 : $243-253$

19 Nissim S, Idos GE, Wu B. Genetic markers of malignant transformation in intraductal papillary mucinous neoplasm of the pancreas: a meta-analysis. Pancreas 2012; 41: 1195 - 1205

20 Distler M, Aust D, Weitz J et al. Precursor lesions for sporadic pancreatic cancer: PanIN, IPMN, and MCN. Biomed Res Int 2014; 2014: 474905

21 Kanda M, Knight S, Topazian $M$ et al. Mutant GNAS detected in duodenal collections of secretin-stimulated pancreatic juice indicates the presence or emergence of pancreatic cysts. Gut 2013; 62: 1024-1033

22 Tan MC, Basturk O, Brannon AR et al. GNAS and KRAS mutations define separate progression pathways in intraductal papillary mucinous neoplasm-associated carcinoma. J Am Coll Surg 2015; 220: 845-854

23 Hosoda W, Sasaki E, Murakami Y et al. GNAS mutation is a frequent event in pancreatic intraductal papillary mucinous neoplasms and associated adenocarcinomas. Virchows Arch 2015; 466: 665-674
24 Singhi AD, Nikiforova MN, Fasanella KE et al. Preoperative GNAS and KRAS testing in the diagnosis of pancreatic mucinous cysts. Clin Cancer Res 2014; 20: 4381 - 4389

25 Amato E, Molin MD, Mafficini A et al. Targeted next-generation sequencing of cancer genes dissects the molecular profiles of intraductal papillary neoplasms of the pancreas. J Pathol 2014; 233: 217-227

26 Bournet B, Souque A, Senesse P et al. Endoscopic ultrasound-guided fine-needle aspiration biopsy coupled with KRAS mutation assay to distinguish pancreatic cancer from pseudotumoral chronic pancreatitis. Endoscopy 2009; 41: $552-557$

27 Bournet B, Selves J, Grand D et al. Endoscopic ultrasound-guided fineneedle aspiration biopsy coupled with a KRAS mutation assay using allelic discrimination improves the diagnosis of pancreatic cancer. J Clin Gastroenterol 2015; 49: 50-56

28 Komatsu H, Tanji E, Sakata $N$ et al. A GNAS mutation found in pancreatic intraductal papillary mucinous neoplasms induces drastic alterations of gene expression profiles with upregulation of mucin genes. PLoS One 2014; 9: e87875

29 Ideno N, Ohtsuka T, Matsunaga $T$ et al. Clinical significance of GNAS mutation in intraductal papillary mucinous neoplasm of the pancreas with concomitant pancreatic ductal adenocarcinoma. Pancreas 2015; 44: $311-320$

30 Aso T, Ohtsuka T, Matsunaga T et al. "High-risk stigmata" of the 2012 international consensus guidelines correlate with the malignant grade of branch duct intraductal papillary mucinous neoplasms of the pancreas. Pancreas 2014; 43: 1239-1243

31 Springer S, Wang Y, Dal Molin M et al. A combination of molecular markers and clinical features improve the classification of pancreatic cysts. Gastroenterology 2015; 149: 1501 - 1510

32 Singhi $A D$, Zeh HJ, Brand RE et al. American Gastroenterological Association guidelines are inaccurate in detecting pancreatic cysts with advanced neoplasia: a clinicopathologic study of 225 patients with supporting molecular data. Gastrointest Endosc 2016; 83: 1107-1117

33 Crippa S, Bassi C, Salvia R et al. Low progression of intraductal papillary mucinous neoplasms with worrisome features and high-risk stigmata undergoing non-operative management: a mid-term follow-up analysis. Gut 2016: pii: DOI 10.1136/gutjnl-2015-310162 